\title{
Bone turnover and periprosthetic bone loss after cementless total hip arthroplasty can be restored by zoledronic acid: a prospective, randomized, open-label, controlled trial
}

Tsan-Wen Huang ${ }^{1,2,7}$, Chao-Jan Wang ${ }^{2,4,6}$, Hsin-Nung Shih ${ }^{2,3,6}$, Yuhan Chang ${ }^{2,3,6}$, Kuo-Chin Huang ${ }^{2,5,8}$, Kuo-Ti Peng ${ }^{1,2,7}$ and Mel S. Lee 2, $^{*}$

\begin{abstract}
Background: Although the loss of bone mineral density (BMD) after total hip arthroplasty (THA) is a known problem, it remains unresolved. This study prospectively examined the effect of zoledronic acid (ZA) on bone turnover and BMD after cementless THA.

Methods: Between January 2010 and August 2011, 60 patients who underwent cementless THA were randomly assigned to receive either ZA infusion or placebo ( $0.9 \%$ normal saline only) postoperatively. ZA was administered at 2 day and 1 year postoperatively. Periprosthetic BMD in seven Gruen zones was assessed preoperatively and at given time points for 2 years. Serum markers of bone turnover, functional scales, and adverse events were recorded.

Results: Each group contained 27 patients for the final analysis. The loss of BMD across all Gruen zones (significantly in zones 1 and 7) up to 2 years postoperatively was noted in the placebo group. BMD was significantly higher in the ZA group than in the placebo group in Gruen zones 1, 2, 6, and 7 at 1 year and in Gruen zones 1, 6, and 7 at 2 years $(p<0.05)$. Compared with baseline measures of BMD, the ZA group had increased BMD in zones $1,2,4,5$, 6 , and 7 at 1 year and in zones $1,4,6$, and 7 at 2 years $(p<0.05)$. Serum bone-specific alkaline phosphatase and $\mathrm{N}$-telopeptide of procollagen I levels were significantly increased at 6 weeks in the placebo group and decreased after 3 months in the ZA group. A transient decrease in osteocalcin level was found at 6 months in the ZA group. Functional scales and adverse events were not different between the two groups.
\end{abstract}

Conclusions: The loss of periprosthetic BMD, especially in the proximal femur (zones 1 and 7), after cementless THA could be effectively reverted using ZA. In addition, bone turnover markers were suppressed until 2 years postoperatively following ZA administration.

Trial registration: Chang Gung Memorial Hospital Protocol Record 98-1150A3, Prevention of Periprosthetic Bone Loss After Total Hip Replacement by Annual Bisphosphonate Therapy, has been reviewed and will be made public on ClinicalTrials.gov. Trial registration number: NCT02838121. Registered on 19 July, 2016.

Keywords: Cementless total hip arthroplasty, Bone mineral density, Bisphosphonate, Stress shielding, Zoledronic acid, Bone turnover markers

\footnotetext{
* Correspondence: bone@doctor.com

${ }^{2}$ Chang Gung University, Taoyuan, Taiwan

${ }^{5}$ Department of Orthopaedic Surgery, Kaohsiung Chang Gung Memorial

Hospital, No. 123, Dapi Rd., Niaosong District, Kaohsiung City 83301, Taiwan

Full list of author information is available at the end of the article
} 


\section{Background}

Cementless total hip arthroplasty (THA) has become popular in recent decades $[1,2]$, but its long-term stability may be limited by progressive bone loss around the prosthetic implant [3, 4]. Periprosthetic bone loss is associated with reduced bone mineral density (BMD) in the periprosthetic Gruen zones [5-7] and may increase the risk of migration, implant loosening, and periprosthetic fractures [8].

A reduction in $\mathrm{BMD}$, especially in the calcar region, is a common sequela of THA [6,9-11]. Thus, preserving bone mineral content is important. Bisphosphonates are anti-resorptive agents that promote bone mineralization and inhibit farnesyl pyrophosphate synthase [12]. Their protective effects after joint arthroplasty have been shown in recent meta-analysis studies [13-15] wherein the periprosthetic BMD continued to increase by $9.40 \%$ at 18-70 months after discontinuation of bisphosphonate therapy.

Zoledronic acid (ZA), a third-generation bisphosphonate, is several times more potent than the first- or second-generation bisphosphonates. It is well tolerated and can rapidly lower bone turnover rates in children and adults at high risk of fractures [16-19]. In the pivotal Health Outcomes and Reduced Incidence with ZA Once-Yearly Trial (HORIZON-PFT), a once-yearly infusion of 5-mg intravenous ZA for a 3-year period significantly reduced the risk of vertebral fractures by $70 \%$, hip fractures by $41 \%$, and non-vertebral fractures by $25 \%$ in post-menopausal women with osteoporosis [20]. ZA protects against osteoporotic fractures [21]. Recently, two studies demonstrated the efficacy of ZA in patients who underwent cementless THA [22, 23], but ZA was administered on different schedules, leaving questions about the optimal dose timing of ZA unanswered.

In the present study, we administered ZA during the early postoperative period after cementless THA, and as a booster dose at 1 year postoperatively. The effects of ZA on periprosthetic BMD and functional outcome measures were assessed prospectively. Additionally, we examined safety concerns surrounding the dosing of ZA.

\section{Methods}

\section{Study design}

This prospective, randomized, open-label clinical trial was registered in ClinicalTrials.gov (NCT02838121). The Institutional Review Board of the study institution approved the study protocol (Reference number 981150A3), which adhered to the Declaration of Helsinki. All of the study participants provided written informed consent.

Based on Arabmotlagh et al. [10], the assumption of mean BMD change was $-8 \%$ in the placebo group and
$6 \%$ in the ZA group, with a standard deviation (SD) of $15 \%$. Power analysis indicated that 25 patients were required, per group, to achieve a power of 0.9 with a $5 \%$ significance level. To avoid drop off and loss to followup, we recruited 30 patients in each group, for a total study sample of 60 patients. The assumptions were proven to be adequate because a similar sample size was reported by another prospective randomized trial [22]. Eligible patients were randomly assigned to either the ZA or placebo groups by an envelope drawing.

On the day following cementless THA, and at 1-year post-THA, the ZA group received $5 \mathrm{mg}$ ZA (Aclasta ${ }^{\oplus}$; Novartis Pharmaceuticals Corporation) via intravenous infusion with $0.9 \%$ normal saline $(500 \mathrm{~mL})$. The control group received only an intravenous saline infusion. All patients received oral calcium (600 $\mathrm{mg}$ ) and vitamin D3 supplements (800 IU) daily throughout the course of the study [24]. Follow-up for radiographic and functional assessments was conducted at 2, 6, and 12 weeks, 6 months, and 1 and 2 years postoperatively.

\section{Patients}

Patients aged 35-85 years undergoing THA, who received dual-energy $\mathrm{x}$-ray absorptiometry (DXA) scanning within the 3 months preceding surgery, were considered for enrollment. Exclusion criteria included use of bisphosphonates during the preceding 2 years; uncontrolled seizure disorders; invasive malignancy within the preceding 5 years; osteogenesis imperfect; multiple myeloma; Paget's disease; iritis; uveitis; diabetic neuropathy/retinopathy; active primary hyperthyroidism; Aspartate aminotransferase (AST), alanine aminotransferase (ALT), or bone-specific alkaline phosphatase levels more than twice the normal limit; serum calcium level >11 mg/dL; hypocalcemia; renal insufficiency (creatinine clearance $\leq 35 \mathrm{~mL} / \mathrm{min}$ ); use of investigational drugs; and the use of hip protectors or implants on the contralateral hip joint.

The patients' demographic data, body mass index, pre-operative diagnoses, and baseline characteristics were recorded.

\section{Total hip arthroplasty}

All of the patients underwent standardized THA via direct lateral approach $[25,26]$ using a Zimmer Trilogy Cup, VerSys Fiber Metal Taper Stem, and highly crosslinked polyethylene, coupled with a $32 \mathrm{~mm}$ metal head. An experienced surgeon performed all of the procedures. Based on our standard of care following cementless THA, the patients were encouraged to ambulate as soon as possible after surgery and advised to protect against weight bearing for 6 weeks. 


\section{Assessments}

An experienced clinician, blinded to group assignment and patients' demographic data, performed all radiographic and clinical assessments.

On each follow-up visit, radiographic evaluation of the total hip prosthesis was performed on each standard antero-posterior views of the pelvis and lateral views of the operated hip according to methods described by Engh et al. [27] and Johnston et al. [28]. The vertical distance between the lateral shoulder of the prosthesis and the superior tip of the greater trochanter on the radiograph was measured. This served as the reference distance for monitoring implant migration. At each study visit, this distance was measured and recorded.

The patients underwent DXA scanning of the operated hip using a densitometer (Hologic Inc., Waltham, MA) for quantifying bone mass and density changes [7]. To estimate the precision of the densitometer, double measurements involving repositioning of the patient and the scanner between the first and second scans were made in 10 patients [29]. The DXA method had a measurement error of $1 \%-3 \%$ in Gruen zones. BMD was measured in the frontal plane, throughout seven Gruen zones, and changes in BMD ratios from baseline were estimated for each zone.

Functional assessments included the Hip Harris Score (HHS), UCLA activity score, Short-Form (SF)-12 Physical Component Summary (PCS), and SF-12 Mental Component Summary (MCS). Renal function (glomerular filtration rate [GFR] and creatinine level), hepatic function (AST and ALT), serum calcium, and levels of bone turnover biomarkers (osteocalcin, bone-specific alkaline phosphatase, and N-telopeptide of procollagen I) were also assessed [30, 31].

Complications, including reported need for analgesics, were recorded. Any medical or surgical event that compromised clinical recovery was defined and recorded as an adverse event. A relatively poorer and slower functional recovery beyond 3 months and an HHS score $<80$ were considered adverse events.

The primary endpoint was the change in periprosthetic BMD, between baseline and all other time points. Secondary endpoints included radiologic analyses, implant migration, levels of serum markers for bone metabolism, functional outcomes, and safety and tolerability of the experimental drug.

\section{Statistical analysis}

Values are presented as mean $( \pm S D)$. Group differences were analyzed using independent-samples $t$-test. Timebased differences were analyzed by repeated measures analysis of variance (ANOVA) using a general linear model with Greenhouse-Geisser correction. Significance was set at $p<0.05$. Post hoc comparisons were performed using Bonferroni corrections for multiple comparisons. SPSS software version 13.0 (SPSS Inc., Chicago, IL) was used for all analyses.

\section{Results}

\section{Patient demographics}

Between January 2010 and August 2011, 60 patients were enrolled. Following randomization into either the ZA or placebo control groups, four patients (one in the ZA group and three in the control group) were excluded due to periprosthetic fractures and an additional two (both from the ZA group) were excluded due to missing BMD data at 1 year (Fig. 1). Each group had 27 patients for the final analysis and no significant differences in baseline characteristics were found between the two groups (Table 1).

\section{Radiographic analysis}

All implants showed stable osteo-integration without evidence of early or late migration. There were no radiolucent lines at the prosthesis-bone interface of the cups and stems, and no pedestal formation in any stem, in either group.

\section{Bone mineral density}

At baseline, both groups had similar BMD. The delta $\mathrm{BMD}$ at each time point revealed that at 12 weeks, the ZA group had significantly higher BMD than the control group in Gruen zones 2, 6, and 7. Increases in BMD persisted at 6 months in zones 6 and 7, at 1 year in zones 1 , 2,6 , and 7 , and at 2 years in zones 1,6 , and 7 (Table 2). The BMD changes from baseline (BMD ratio) were significantly higher for the ZA group in Gruen zones 2, 4,6 , and 7 at 12 weeks; in zones 1, 6, and 7 at 6 months; in zones 1, 2, 4, 5, 6 and 7 at 1 year, and in zones 1, 4, 6, and 7 at 2 years (Table 3 ).

Time-based BMD differences in each Gruen zones were analyzed by repeated measure ANOVA (Fig. 2). In zone 1, the mean BMD change was $111 \%$ in the ZA group and $88 \%$ in the control group (95\% CI, 10\%-36\%; $p=0.001)$. In zone 2 , the mean BMD change was $114 \%$ (ZA) and 103\% (control) (95\% CI, 2\%-20\%; $p=0.018$ ). In zone 4 , the mean BMD change was $105 \%(\mathrm{ZA})$ and $95 \%$ (control) (95\% CI, 5\%-15\%; $p=0.001)$. In zone 5 , the mean BMD change was $108 \%(\mathrm{ZA})$ and $99 \%$ (control) (95\% CI, $1 \%-18 \% ; p=0.024)$. In zone 6 , the mean BMD change was 114\% (ZA) and 98\% (control) (95\% CI, $7 \%-25 \% ; p=0.001)$. In zone 7 , the mean BMD change was $110 \%$ (ZA) and $84 \%$ (control) (95\% CI, $14 \%-37 \% ; p<0.001)$.

\section{Functional outcomes}

There were no significant differences in HHS, SF-12 (PCS), SF-12 (MCS), and UCLA scores between the 


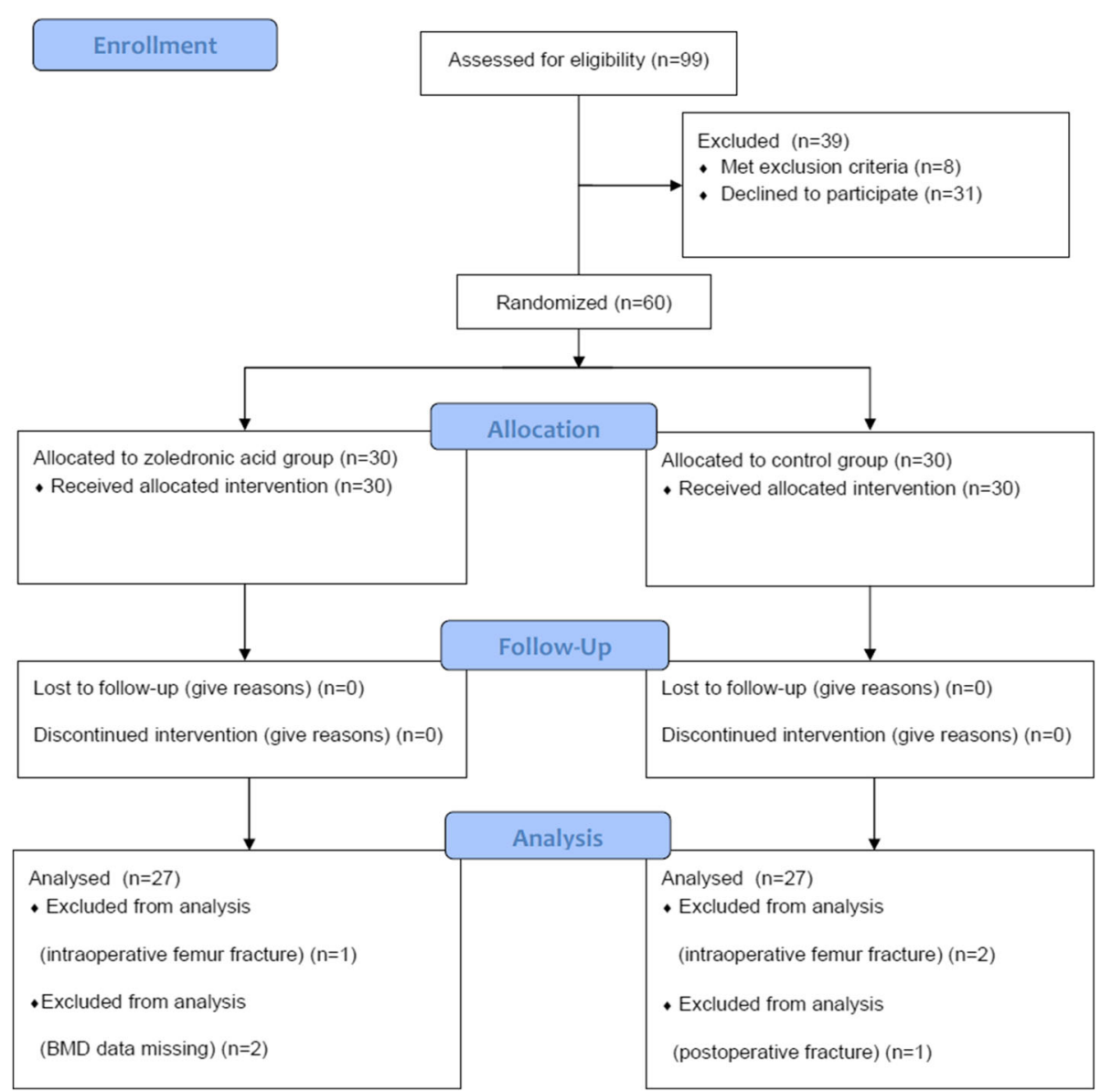

Fig. 1 Patient disposition

groups at any point in the study. However, the withingroup functional scores changed significantly throughout the study period $(p<0.001)$ (Table 4$)$. HHS increased significantly from baseline to 6 weeks, and thereafter up to 2 years, in both groups $(p<0.001)$. SF-12 (PCS) scores were lower at 2 weeks compared to baseline $(p<0.001)$, but increased significantly from baseline to 12 weeks, and thereafter up to 2 years, in both groups $(p<0.001)$.

Table 1 Patient demographics and surgical results

\begin{tabular}{lll}
\hline & $\begin{array}{l}\text { ZA group } \\
(n=27)\end{array}$ & $\begin{array}{l}\text { Control group } \\
(n=27)\end{array}$ \\
\hline Age (years), mean (SD) & $60.1(11.7)$ & $59.4(13.3)$ \\
Sex, female:male & $15: 12$ & $14: 13$ \\
BMI (kg/m²), mean (SD) & $26(4)$ & $25(5)$ \\
$\begin{array}{l}\text { Operation time (minutes), } \\
\text { mean (SD) }\end{array}$ & $118(27)$ & $103(17)$ \\
Blood loss (mL), mean (SD) & $502(312)$ & $387(162)$ \\
Diagnosis (OA/AVN) & $16 / 11$ & $17 / 10$ \\
\hline BMI body mass index SD standat
\end{tabular}

$B M I$ body mass index, $S D$ standard deviation, $Z A$ zoledronic acid, $O A$ osteoarthritis, $A V N$ avascular necrosis

${ }^{a}$ statistically significant difference between the ZA and control groups $(P<0.05)$
The SF-12 (MCS) scores of both groups were significantly lower at 1 year compared to 6 weeks $(p<0.05)$. Compared to baseline, the UCLA scores of both groups were significantly lower at 2 and 6 weeks $(p<0.01)$, but significantly increased by 1 year $(p<0.001)$.

\section{Renal and hepatic function and serum calcium levels}

There were no significant differences in creatinine levels between the two groups at baseline or at any time point throughout the study. GFR increased within both groups between baseline and 6 months $(p<0.01)$ and baseline and 1 year $(p<0.001)$ (Table 5$)$. The AST and ALT values at baseline and at 6 and 12 weeks were within normal limits and had no group differences were realized at any time point. Serum calcium levels were similar in both groups at all time points.

\section{Biomarkers of bone turnover}

There was a significant reduction, from baseline, in levels of bone-specific alkaline phosphatase for the ZA group at 12 weeks, 6 months, 1 year, and 2 years postoperatively $(p<0.01)$. In the control group, bone-specific 
Table 2 Mean (SD) BMD $\left(\mathrm{g} / \mathrm{cm}^{2}\right)$ for both groups in all gruen zones

\begin{tabular}{|c|c|c|c|c|c|c|c|c|}
\hline Gruen zones & 1 & 2 & 3 & 4 & 5 & 6 & 7 & Delta \\
\hline \multicolumn{9}{|l|}{ Baseline } \\
\hline ZA group & $0.64(0.14)$ & $1.26(0.21)$ & $1.52(0.24)$ & $1.63(0.19)$ & $1.57(0.19)$ & $1.24(0.21)$ & $0.96(0.19)$ & 1.15(0.16) \\
\hline Control & $0.64(0.16)$ & $1.24(0.22)$ & $1.53(0.21)$ & $1.66(0.21)$ & $1.61(0.23)$ & $1.25(0.22)$ & $0.97(0.24)$ & $1.15(0.17)$ \\
\hline$p$-value & 0.863 & 0.778 & 0.779 & 0.522 & 0.446 & 0.951 & 0.830 & 0.968 \\
\hline \multicolumn{9}{|l|}{12 weeks } \\
\hline ZA group & $0.74(0.38)$ & $1.46(0.28)$ & $1.69(0.88)$ & $1.73(0.35)$ & $1.75(0.58)$ & $1.40(0.21)$ & $1.09(0.22)$ & $1.38(0.60)$ \\
\hline Control & $0.59(0.14)$ & $1.28(0.21)$ & $1.50(0.21)$ & $1.61(0.21)$ & $1.61(0.22)$ & $1.23(0.23)$ & $0.88(0.20)$ & $1.16(0.14)$ \\
\hline$p$-value & 0.060 & $0.010^{\mathrm{a}}$ & 0.276 & 0.142 & 0.238 & $0.009^{\mathrm{a}}$ & $0.001^{\mathrm{a}}$ & 0.077 \\
\hline \multicolumn{9}{|l|}{6 months } \\
\hline ZA group & $0.65(0.14)$ & $1.40(0.17)$ & $1.55(0.17)$ & 1.68(0.19) & $1.62(0.27)$ & $1.40(0.19)$ & $1.04(0.25)$ & $1.26(0.12)$ \\
\hline Control & $0.59(0.15)$ & $1.31(0.26)$ & $1.51(0.22)$ & $1.62(0.23)$ & $1.63(0.23)$ & $1.26(0.23)$ & $0.84(0.30)$ & $1.17(0.16)$ \\
\hline$p$-value & 0.109 & 0.144 & 0.529 & 0.301 & 0.922 & $0.021^{\mathrm{a}}$ & $0.008^{\mathrm{a}}$ & $0.021^{\mathrm{a}}$ \\
\hline \multicolumn{9}{|l|}{1 year } \\
\hline ZA group & $0.66(0.15)$ & $1.38(0.19)$ & $1.50(0.24)$ & 1.68(0.19) & 1.66(0.16) & $1.40(0.22)$ & $1.01(0.27)$ & $1.25(0.13)$ \\
\hline Control & $0.54(0.20)$ & $1.19(0.38)$ & $1.44(0.38)$ & $1.54(0.38)$ & $1.51(0.39)$ & $1.20(0.36)$ & $0.80(0.30)$ & $1.11(0.28)$ \\
\hline$p$-value & $0.021^{\mathrm{a}}$ & $0.035^{a}$ & 0.434 & 0.096 & 0.057 & $0.014^{\mathrm{a}}$ & $0.011^{a}$ & $0.021^{a}$ \\
\hline \multicolumn{9}{|l|}{2 years } \\
\hline ZA group & $0.67(0.15)$ & $1.38(0.20)$ & $1.49(0.25)$ & $1.68(0.19)$ & $1.65(0.20)$ & $1.44(0.21)$ & $1.01(0.27)$ & $1.26(0.13)$ \\
\hline Control & $0.55(0.15)$ & $1.35(0.77)$ & $1.51(0.23)$ & $1.59(0.23)$ & $1.62(0.21)$ & $1.21(0.24)$ & $0.78(0.22)$ & $1.14(0.15)$ \\
\hline$p$-value & $0.013^{a}$ & 0.870 & 0.786 & 0.167 & 0.612 & $0.001^{a}$ & $0.003^{a}$ & $0.011^{a}$ \\
\hline
\end{tabular}

${ }^{a}$ statistically significant difference between ZA and control groups

$B M D$ bone mineral density, SD standard deviation, ZA zoledronic acid

alkaline phosphatase was significantly increased at 6 and 12 weeks postoperatively $(p<0.05)$ (Table 5$)$. In the ZA group, osteocalcin was significantly reduced between baseline and 6 months, 1 year, and 2 years $(p<0.05)$. The control group demonstrated significant increases in osteocalcin between baseline and 1 year $(p<0.05)$.

In the ZA group, levels of N-telopeptide of procollagen I were significantly reduced from baseline at 12 weeks, 6 months, 1 year, and 2 years postoperatively

Table 3 Mean (SD) bone mineral density ratio (the BMD changes from baseline) for each group by gruen zone at different time points

\begin{tabular}{|c|c|c|c|c|c|c|c|}
\hline Gruen zones & 1 & 2 & 3 & 4 & 5 & 6 & 7 \\
\hline \multicolumn{8}{|l|}{12 weeks } \\
\hline ZA group & $1.20(0.74)$ & $1.18(0.28)^{a}$ & $1.13(0.62)$ & $1.07(0.23)^{a}$ & $1.13(0.41)$ & $1.13(0.13)^{a}$ & $1.15(0.21)^{\mathrm{a}}$ \\
\hline Control & $0.93(0.14)$ & $1.04(0.12)$ & 0.98(0.08) & $0.97(0.04)$ & $1.00(0.07)$ & $1.00(0.15)$ & $0.93(0.22)$ \\
\hline \multicolumn{8}{|l|}{6 months } \\
\hline ZA group & $1.05(0.19)^{a}$ & $1.13(0.12)$ & $1.05(0.21)$ & $1.04(0.07)$ & $1.04(0.18)$ & $1.14(0.12)^{a}$ & $1.09(0.20)^{\mathrm{a}}$ \\
\hline Control & $0.92(0.18)$ & $1.07(0.19)$ & 0.99(0.08) & $0.97(0.06)$ & $1.01(0.07)$ & $1.02(0.18)$ & $0.88(0.29)$ \\
\hline \multicolumn{8}{|l|}{1 year } \\
\hline ZA group & $1.05(0.19)^{a}$ & $1.10(0.10)^{a}$ & $1.00(0.09)$ & $1.03(0.08)^{a}$ & $1.07(0.12)^{a}$ & $1.13(0.13)^{a}$ & $1.07(0.22)^{a}$ \\
\hline Control & $0.86(0.24)$ & $0.98(0.25)$ & $0.95(0.22)$ & $0.93(0.20)$ & $0.95(0.24)$ & $0.97(0.28)$ & $0.84(0.29)$ \\
\hline \multicolumn{8}{|l|}{2 years } \\
\hline ZA group & $1.08(0.21)^{a}$ & $1.12(0.11)$ & $1.00(0.11)$ & $1.04(0.09)^{a}$ & $1.08(0.15)$ & $1.16(0.13)^{a}$ & $1.06(0.22)^{a}$ \\
\hline Control & $0.89(0.14)$ & $1.10(0.46)$ & $1.00(0.08)$ & $0.96(0.07)$ & $1.02(0.10)$ & $1.00(0.19)$ & $0.83(0.20)$ \\
\hline
\end{tabular}






Gruen 5

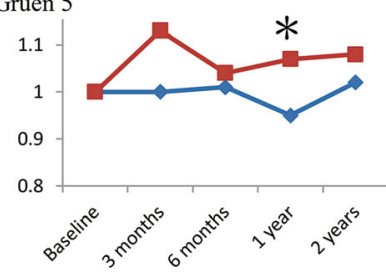

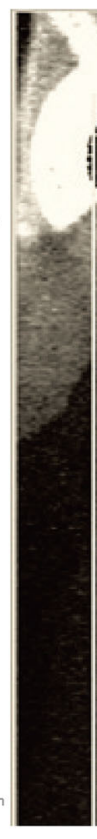

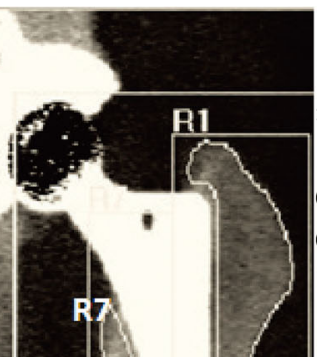

Gruen 1
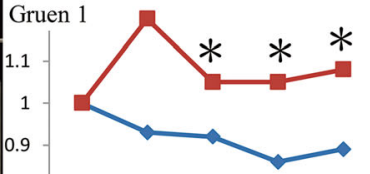

0.8


R2

Gruen 2

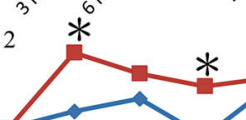

A5.
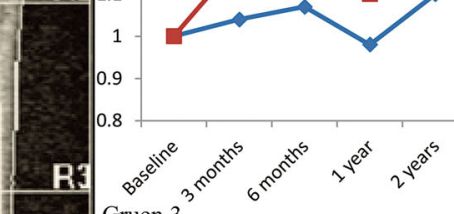

Gruen 3

1.1



0.8

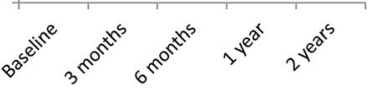

$\rightarrow$ Control $\rightarrow$-Zoledronic acid

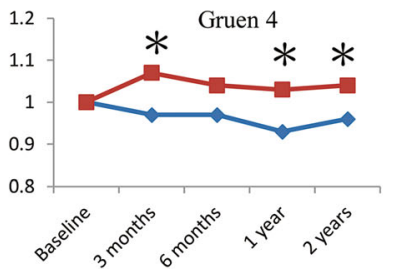

Fig. 2 Bone mineral density changes in the zoledronic acid and control groups in Gruen zones

Table 4 Clinical assessments in each group at different time intervals $(n=54)$

\begin{tabular}{|c|c|c|c|c|c|c|c|c|c|c|c|c|c|c|}
\hline & \multicolumn{2}{|c|}{ Preop } & \multicolumn{2}{|c|}{2 week } & \multicolumn{2}{|c|}{6 week } & \multicolumn{2}{|c|}{3 months } & \multicolumn{2}{|c|}{6 months } & \multicolumn{2}{|c|}{1 year } & \multicolumn{2}{|c|}{2 years } \\
\hline & $\mathrm{N}$ & mean $\pm S D$ & $\mathrm{~N}$ & mean \pm SD & $\mathrm{N}$ & mean $\pm S D$ & $\mathrm{~N}$ & mean $\pm S D$ & $\mathrm{~N}$ & mean \pm SD & $\mathrm{N}$ & mean $\pm S D$ & $\mathrm{~N}$ & mean $\pm S D$ \\
\hline \multicolumn{15}{|c|}{ Harris hip score } \\
\hline Group ZA & 27 & $60.31 \pm 10.49$ & 27 & $56.23 \pm 14.06$ & 27 & $70.96 \pm 13.03^{a}$ & 27 & $77.93 \pm 10.63^{a}$ & 27 & $83.60 \pm 7.52^{a}$ & 27 & $86.34 \pm 7.08^{a}$ & 27 & $90.18 \pm 2.11^{a}$ \\
\hline Group N/S & 27 & $60.88 \pm 11.63$ & 27 & $58.80 \pm 10.13$ & 27 & $71.08 \pm 12.26^{\mathrm{a}}$ & 27 & $79.89 \pm 9.42^{a}$ & 27 & $81.50 \pm 9.87^{a}$ & 27 & $85.16 \pm 7.28^{a}$ & 27 & $87.67 \pm 5.44^{a}$ \\
\hline \multicolumn{15}{|l|}{ SF-12(PCS) } \\
\hline Group ZA & 27 & $27.53 \pm 10.13$ & 27 & $18.96 \pm 7.48^{a}$ & 27 & $28.23 \pm 11.35$ & 27 & $40.01 \pm 13.54^{a}$ & 27 & $48.09 \pm 6.95^{a}$ & 27 & $51.29 \pm 4.45^{\mathrm{a}}$ & 27 & $53.69 \pm 3.79^{a}$ \\
\hline Group N/S & 27 & $29.16 \pm 11.31$ & 27 & $21.37 \pm 8.34^{\mathrm{a}}$ & 27 & $27.79 \pm 11.99$ & 27 & $42.12 \pm 10.61^{a}$ & 27 & $48.96 \pm 7.90^{a}$ & 27 & $50.53 \pm 6.95^{a}$ & 27 & $52.50 \pm 4.16^{a}$ \\
\hline \multicolumn{15}{|l|}{ SF-12(MCS) } \\
\hline Group ZA & 27 & $57.65 \pm 12.57$ & 27 & $60.87 \pm 9.51$ & 27 & $59.23 \pm 10.59$ & 27 & $60.3 \pm 6.86$ & 27 & $58.85 \pm 4.95$ & 27 & $58.49 \pm 4.07$ & 27 & $58.61 \pm 5.98$ \\
\hline Group N/S & 27 & $59.17 \pm 11.99$ & 27 & $60.08 \pm 7.29$ & 27 & $63.72 \pm 3.84$ & 27 & $59.9 \pm 4.77$ & 27 & $55.97 \pm 8.34$ & 27 & $56.96 \pm 7.20$ & 27 & $59.01 \pm 4.05$ \\
\hline \multicolumn{15}{|c|}{ UCLA activity score } \\
\hline Group ZA & 27 & $3.96 \pm 1.16$ & 27 & $2.56 \pm 0.80^{a}$ & 27 & $3.41 \pm 0.97^{\mathrm{a}}$ & 27 & $4.22 \pm 0.93$ & 27 & $4.63 \pm 1.15$ & 27 & $5.74 \pm 1.06^{a}$ & 27 & $7.00 \pm 0.90^{a}$ \\
\hline Group N/S & 27 & $4.33 \pm 1.59$ & 27 & $2.56 \pm 0.70^{a}$ & 27 & $3.44 \pm 0.97^{a}$ & 27 & $4.52 \pm 0.94$ & 27 & $4.70 \pm 0.95$ & 27 & $5.52 \pm 1.19^{a}$ & 27 & $6.50 \pm 0.74^{a}$ \\
\hline
\end{tabular}

Statistically significant difference between ZA and control groups

${ }^{a}$ significant difference between each time point and baseline

SD Standard deviation, ZA zoledronic acid, SF short form, PCS physical component summary, MCS mental component summary, N/S normal saline 
Table 5 Mean serum creatinine (Cr) level, glomerular filtration rate (GFR), and bone turnover biomarkers in the ZA (zoledronic acid) and control groups as a function of time

\begin{tabular}{|c|c|c|c|c|c|c|}
\hline & Baseline & 6 weeks & 12 weeks & 6 months & 1 year & 2 years \\
\hline \multicolumn{7}{|l|}{ Renal function } \\
\hline \multicolumn{7}{|l|}{$\operatorname{Cr}(m g / d L)$} \\
\hline ZA group & $0.79(0.23)$ & $0.76(0.27)$ & $0.81(0.28)$ & $0.85(0.59)$ & $0.78(0.24)$ & $0.76(0.26)$ \\
\hline Control & $0.83(0.2)$ & $0.81(0.22)$ & $0.79(0.18)$ & $0.79(0.19)$ & $0.76(0.23)$ & $0.79(0.22)$ \\
\hline \multicolumn{7}{|l|}{ GFR (mL/min) } \\
\hline ZA group & $59.39(2.21)$ & $60.08(5)$ & $61.84(10.78)$ & $77.56(27.24)$ & $83.8(28.48)$ & $93.9(23.3)$ \\
\hline Control & $59.63(1.28)$ & $59.78(1.15)$ & $63.16(12.83)$ & $73.82(23.6)$ & $79.45(25.96)$ & $87.3(19.5)$ \\
\hline \multicolumn{7}{|l|}{$\mathrm{Ca}(m g / d L)$} \\
\hline ZA group & $9.53(0.49)$ & $9.34(0.57)$ & $9.52(0.44)$ & $9.46(0.46)$ & $9.43(0.49)$ & $9.37(0.39)$ \\
\hline Control & $9.57(0.43)$ & $9.2(1.77)$ & $9.54(0.36)$ & $9.47(0.52)$ & $9.13(1.89)$ & $9.24(0.29)$ \\
\hline \multicolumn{7}{|c|}{ Bone turnover biomarkers } \\
\hline \multicolumn{7}{|c|}{ Bone-specific alkaline phosphatase $(\mu \mathrm{g} / \mathrm{L})$} \\
\hline ZA group & $78.6(17.7)$ & $77.3(19.2)^{\mathrm{a}}$ & $65.6(11.7)^{\mathrm{b}}$ & $67.5(26.6)$ & $68.7(24.5)$ & $66.5(18.1)$ \\
\hline Control & $76(17.6)$ & $89.4(21.7)$ & $83.3(22.8)$ & $74.5(19.9)$ & $73.2(22.9)$ & $74(15.9)$ \\
\hline \multicolumn{7}{|c|}{ Osteocalcin (ng/mL) } \\
\hline ZA group & $21.1(8.7)$ & $18.1(7.9)$ & $17.2(10.2)$ & $14.7(5)$ & $16.8(7.2)$ & $14.9(7.2)$ \\
\hline Control & $18.5(10.3)$ & $20.9(13.2)$ & $20.8(11.5)$ & $17.7(6.4)$ & $20.5(10.4)$ & $18.8(8.6)$ \\
\hline \multicolumn{7}{|c|}{ N-telopeptide of procollagen I ( $\mathrm{ng} / \mathrm{mL})$} \\
\hline ZA group & $53.9(23.1)$ & $57.1(24.6)^{\mathrm{a}}$ & $38.8(16.9)^{b}$ & $30.5(11.9)^{b}$ & $34.6(19.5)$ & $28.3(11.3)^{\mathrm{a}}$ \\
\hline Control & $43.2(21.8)$ & $80.7(44)$ & $69.7(38)$ & $57.5(24.9)$ & $50.4(35.4)$ & $44.3(19.3)$ \\
\hline
\end{tabular}

asignificant difference between groups $(P<0.05)$

${ }^{\mathrm{b}}$ significant difference between groups $(P<0.001)$

$(p<0.05)$. In the control group, these levels significantly increased from baseline at 6 weeks, 12 weeks, and at 6 months postoperatively $(p<0.05)$. There were significant differences in bone turnover biomarkers between the ZA and control groups (Table 5).

\section{Adverse events}

Complications included fever $(n=3)$ and hypocalcemia $(n=1)$ but were mild-to-moderate in severity, and believed to be related to the investigational drug. No patient sustained osteonecrosis of the jaw or atypical femoral fracture. Three patients (one in the ZA group and two in the control group) had intra-operative periprosthetic fractures, and one (control) subject had postoperative periprosthetic fracture. These individuals were excluded from the final analysis.

\section{Discussion}

The present study shows that ZA infusions on the day after cementless THA, and as a booster at 1 year postoperatively, significantly reduced periprosthetic femoral BMD loss. In the control group, BMD decreased significantly in Gruen zones 1 and 7 up to 2 years postoperatively. In contrast, for the ZA group, BMD significantly increased in all Gruen zones (except for zone 3) between baseline and 1 year postoperatively. At 2 years postoperatively it remained significantly increased in zones 1,6 , and 7. The greatest effect of ZA was observed in the proximal femur at 2 years, with BMD changes of $+6 \%$ (control: $-17 \%$ ) in zone 7 and $+8 \%$ (control: $-11 \%$ ) in zone 1 . However, this improvement does not necessarily reflect better functional outcomes.

Only two reports have previously studied ZA in patients after cementless THA. Scott et al. [23] reported that ZA significantly prevented BMD loss in Gruen zones 1,4 , and 6 at 1 year, and in zones 1 and 6 at 2 years. However a decrease in BMD was still evident in zone 7 (calcar region) at 1 and 2 years. Our results were different. We found increased BMD after ZA across all Gruen zones at 1 year (though this difference did not rise to the level of statistical significance for zone 3), including Gruen zone 7. We thought the difference may be related to differences in Scott et al.'s timing of ZA administration ( 2 weeks postoperatively), or differences among the various types of prosthesis (two types of modular stems). The present study always administered $\mathrm{ZA}$ at 2 days postoperatively and used only one type of prosthesis. Periprosthetic BMD varies as a function of stem type. Patients with large and stiff femoral stems experience greater stress shielding, which results in more 
resorption of the proximal femur [15]. The modular femoral prosthesis has a larger proximal implant segment (for the neck-body taper junction) and is stiffer proximally. Proximal stiffness mismatches may increase proximal femoral stress shielding and subsequent bone loss. Moreover, more proximal bone loss occurs in femur preparation for larger femoral prosthesis. A femur with less bone mass is less stiff, relative to the implanted stem, and this will increase stress-related bone resorption [32]. In this study, we used a flat and double-tapered nonmodular femoral stems. This design decreases the crosssectional area moment of inertia and achieves initial stability by wedging into the proximal femur. It is considerably less stiff than the modular design and prevents periprosthetic bone loss.

In the second study, ZA (4 mg) was administered on the first postoperative day to 25 patients after cementless THA. Periprosthetic BMD was not analyzed but ZA improved the initial fixation of the cementless implant and prevented early implant migration, compared to 24 control patients [22]. The current study also used ZA in the acute postoperative period and did not find severe adverse events related to the drug. ZA infusion was deemed safe during the acute phase after THA. Taken together, early timing for postoperative ZA treatment may be a safe and effective means of preventing BMD loss after cementless THA.

Since periprosthetic bone loss is most pronounced in the early postoperative period [9], administering bisphosphonates soon after surgery is logical [22]. Ericksen and colleagues reported that the timing of the first ZA infusion changes BMD for patients who recently underwent hip surgery [33]. At 1 year postoperatively, all patients treated with ZA had increased BMD except for the group that received an early dose ( $\leq 2$ weeks postoperatively). This group also demonstrated worse anti-fracture outcomes. While the current findings seem to contradict those of Ericksen et al., the patients in their study who received ZA $\leq 2$ weeks after surgery had a higher risk of mortality due to older age and a greater number of comorbidities. Moreover, their study had a smaller sample size and therefore group heterogeneity may have affected their findings. The current study provides a consolidated examination of BMD and revealed biochemical data supporting the use of ZA in the early postoperative period ( 2 days postoperatively). Since THA or bipolar hemiarthroplasty are common surgeries for patients with hip fractures, early initiation of ZA or other bisphosphonates is practical and may lead to superior outcomes.

In their meta-analysis, Bhandari et al. [13] suggested that bisphosphonates had a beneficial effect in maintaining periprosthetic BMD after THA or total knee arthroplasty. Arabmotlagh et al. [10] reported longstanding beneficial effects of alendronate in the prevention of periprosthetic bone loss 6 years after cementless THA. A single-dose of ZA can restore BMD beyond 1 year, with an effect comparable to that obtained with three annual ZA infusions [34]. In addition, ZA can prevent bone loss for long time periods. The HORIZON-PFT study reports a $6.02 \%$ increase in total hip BMD in patients, at risk of hip fractures, following 3 years of annual ZA treatment $[19,35]$. Improvements in BMD continued for 6 years in these patients, suggesting a lasting effect of ZA.

Measuring serum calcium levels during ZA treatment is recommended [23]. In this study, both groups were administered oral calcium supplements. Serum calcium levels were within normal range throughout the study period (Table 5). Since ZA can lead to reduced serum calcium levels 6 weeks after its administration, providing calcium supplements is imperative.

Previous studies report short-term increases in creatinine after treatment, which typically resolve quickly, and without long-term impact on renal function [35]. In this study, there were no significant differences in renal function between the ZA and control groups, supportive of improved GFR over time.

This study found that bone turnover markers were significantly altered by the administration of ZA. Decreased bone turnover markers may contribute to the development of complications such as bisphosphonate-induced osteonecrosis of the jaw (ONJ) $[23,36]$ or atypical femoral fractures [37, 38]. ZA was administered over two doses in this study. This dosing was different from past studies of fracture prevention or studies that reported on complications such as $\mathrm{ONJ}$ and atypical femoral fractures related to prolonged use of bisphosphonates. Extending the current study period will be beneficial since our data revealed changes in bone turnover markers toward the conclusion of the study. By following patients for more than 2 years, we may learn more about these changes, as well as the risks of bisphosphonate-related complications.

The main limitation of this study was the sample size. Nonetheless, the findings are compelling and consistent with those of previous reports $[12,18,20]$. Although this study was powered to show changes in BMD throughout various Gruen zones, at various time points postoperatively, some zones exhibited borderline significances. It is possible that the study was underpowered and a larger sample size may be needed to demonstrate significant changes in BMD following administration of ZA. Bone mineral homeostasis correlates with the calcium and vitamin D metabolism. Another limitation of the current study was our failure to measure vitamin D levels at baseline. Although all study subjects had normal BMDs, labs, and no osteoporosis, we cannot rule out the possibility of an underlying vitamin D deficiency in study 
subjects. A future studies should clarify the effects of vitamin D levels.

\section{Conclusions}

In conclusion, initiation of $\mathrm{ZA}$ treatment in the acute postoperative period preserves periprosthetic $\mathrm{BMD}$ in the proximal femur (zones 1 and 7). ZA may be effective prophylaxis against periprosthetic bone loss and implant migration. Future large-scale, longitudinal studies are necessary to demonstrate clinical effectiveness and investigate the risk of treatment-related complications.

\section{Abbreviations}

ALT: Alanine aminotransferase; ANOVA: Analysis of variance; AST: Aspartate aminotransferase; BMD: Bone mineral density; DXA: Dual-energy x-ray absorptiometry; GFR: Glomerular filtration rate; HHS: Hip Harris Score; MCS: Mental Component Summary; PCS: Physical Component Summary: SD: Standard deviation; SF-12: Short-Form-12; THA: Total hip arthroplasty; ZA: Zoledronic acid

\section{Acknowledgments}

We thank Ms. Yu-Shuan Lin for recording radiographic and functional data and for assisting with the statistical analyses.

\section{Funding}

This study (CZOL446HTW07T study) was sponsored by Novartis. No additional grant support was provided.

\section{Availability of data and materials}

The dataset supporting the conclusions of this article is included within the article. Participants did not consent to public release of their data. However data can be made available on request for researchers who meet the criteria for access to confidential data from the Department of Orthopaedic Surgery, Chang Gung Memorial Hospital, Chiayi, Taiwan.

\section{Authors' contribution}

MSL and H-NS contributed substantially to the conception and design of the study. C-JW, YC, K-CH, and K-TP analyzed and interpreted the data. T-WH drafted the article and revised it critically for important intellectual content. All authors reviewed and edited the manuscript and approved the final version of the manuscript.

\section{Competing interests}

All authors declare that they have no competing interests.

\section{Consent for publication}

Consents regarding individual details, images, and videos have been obtained from all patients.

\section{Ethics approval and consent to participate}

This prospective, randomized, open-label, single-center clinical trial with 1:1 allocation was registered in the public registry ClinicalTrials.gov (Reference number NCT02838121). The Institutional Review Board of the study institution approved the study protocol (Reference number 98-1150A3), and all patients provided signed informed consent.

\section{Publisher's Note}

Springer Nature remains neutral with regard to jurisdictional claims in published maps and institutional affiliations,

\section{Author details}

'Department of Orthopaedic Surgery, Chang Gung Memorial Hospital, Chiayi, Taiwan. ${ }^{2}$ Chang Gung University, Taoyuan, Taiwan. ${ }^{3}$ Department of Orthopaedic Surgery, Chang Gung Memorial Hospital, Linkou, Taiwan. ${ }^{4}$ Department of Diagnostic and Interventional Radiology, Chang Gung Memorial Hospital, Linkou, Taiwan. ${ }^{5}$ Department of Orthopaedic Surgery, Kaohsiung Chang Gung Memorial Hospital, No. 123, Dapi Rd., Niaosong
District, Kaohsiung City 83301, Taiwan. ${ }^{6} 5$ Fu-Hsin Street, Kweishan, Taoyuan, Taiwan. 'No. 6, West Section, Chia-Pu Road, Pu-Tzi City, Chia-Yi Hsien 613, Taiwan. ${ }^{8}$ No. 123, Dapi Rd., Niaosong District, Kaohsiung City 83301, Taiwan.

Received: 15 February 2017 Accepted: 12 May 2017

Published online: 22 May 2017

\section{References}

1. Stephen R, Burnett J. Total hip arthroplasty: techniques and results. BCMJ. 2010;52:455-64

2. Markel DC, Hoard DB, Porretta CA. Cemented total hip arthroplasty with Boneloc bone cement. J South Orthop Assoc. 2001;10:202-8.

3. Chen $\mathrm{G}$, Shukeir N, Potti A, et al. Up-regulation of Wnt-1 and beta-catenin production in patients with advanced metastatic prostate carcinoma: potential pathogenetic and prognostic implications. Cancer. 2004;101:1345-56.

4. Yamada H, Yoshihara Y, Henmi O, et al. Cementless total hip replacement: past, present, and future. J Orthop Sci. 2009;14:228-41.

5. Oh I, Harris WH. Proximal strain distribution in the loaded femur. An in vitro comparison of the distributions in the intact femur and after insertion of different hip-replacement femoral components. J Bone Joint Surg Am. 1978;60:75-85.

6. Alm JJ, Makinen TJ, Lankinen P, et al. Female patients with low systemic BMD are prone to bone loss in Gruen zone 7 after cementless total hip arthroplasty. Acta Orthop. 2009:80:531-7.

7. Iolascon G, Di Pietro G, Capaldo A, et al. Peri-prosthetic bone density as outcome of therapeutic response. Clin Cases Miner Bone Metab. 2010;7:27-31

8. Aro HT, Alm JJ, Moritz N, et al. Low BMD affects initial stability and delays stem osseointegration in cementless total hip arthroplasty in women: a 2year RSA study of 39 patients. Acta Orthop. 2012;83:107-14.

9. Venesmaa PK, Kröger HP, Miettinen HJ, et al. Monitoring of peri-prosthetic BMD after uncemented total hip arthroplasty with dural-energy X-ray absorptiometry - a 3-year follow-up study. J Bone Miner Res. 2001;16:1056-61.

10. Arabmotlagh M, Pilz M, Warzecha J, et al. Changes of femoral periprosthetic bone mineral density 6 years after treatment with alendronate following total hip arthroplasty. J Orthop Res. 2009;27:183-8.

11. Smolders JM, Hol A, Rijnders T, et al. Changes in bone mineral density in the proximal femur after hip resurfacing and uncemented total hip replacement: A prospective randomised controlled study. J Bone Joint Surg (Br). 2010;92:1509-14.

12. Ott SM. What is the optimal duration of bisphosphonate therapy? Cleve Clin Jed. 2011;78:619-30.

13. Bhandari M, Bajammal S, Guyatt GH, et al. Effect of bisphosphonates on periprosthetic bone mineral density after total joint arthroplasty. A meta-analysis. J Bone Joint Surg Am. 2005;87:293-301.

14. Lin T, Yan SG, Cai XZ, et al. Bisphosphonates for peri-prosthetic bone loss after joint arthroplasty: a meta-analysis of 14 randomized controlled trials. Osteoporos Int. 2012;23:1823-34.

15. Knusten AR, Ebramzadeh E, Longjohn DB, et al. Systematic analysis of bisphosphonate intervention on periprosthetic BMD as a function of stem design. J Arthroplasty. 2014;29:1292-7.

16. Yoshimura M, Kohno N, Yamada K, et al. (89)Sr imaging with bremsstrahlung in patients with metastatic breast cancer. Clin Nucl Med. 2012;37:1035-40.

17. Rakel A, Boucher A, Ste-Marie LG. Role of zoledronic acid in the prevention and treatment of osteoporosis. Clin Interv Aging. 2011;6:89-99.

18. Simm PJ, Johannesen J, Briody J, et al. Zoledronic acid improves bone mineral density, reduces bone turnover and improves skeletal architecture over 2 years of treatment in children with secondary osteoporosis. Bone. 2011:49:939-43.

19. Orwoll ES, Miller PD, Adachi JD, et al. Efficacy and safety of a once-yearly i.v. Infusion of zoledronic acid 5 mg versus a once-weekly 70-mg oral alendronate in the treatment of male osteoporosis: a randomized, multi-center, doubleblind, active-controlled study. J Bone Miner Res. 2010:25·2239-50.

20. Black DM, Delmas PD, Eastell R, et al. HORIZON Pivotal Fracture Trial, Onceyearly zoledronic acid for treatment of post-menopausal osteoporosis. N Engl J Med. 2007;356:1809-22.

21. Jansen JP, Bergman GJ, Huels J, et al. The efficacy of bisphosphonates in the prevention of vertebral, hip, and non-vertebral-nonhip fractures in osteoporosis: a network meta-analysis. Semin Arthritis Rheum. 2011;40:275-84. e1-2 
22. Friedl G, Radl R, Stihsen C, et al. The effect of a single infusion of zoledronic acid on early implant migration in total hip arthroplasty. A randomized, double-blind, controlled trial. J Bone Joint Surg Am. 2009;91:274-81.

23. Scott DF, Woltz JN, Smith RR. Effect of Zoledronic acid on reducing femoral bone mineral density loss following total hip arthroplasty: preliminary results of a prospective randomized trial. J Arthroplasty. 2013;28:671-5.

24. Lambrinoudaki I, Vlachou S, Galapi F, et al. Once-yearly zoledronic acid in the prevention of osteoporotic bone fractures in post-menopausal women. Clin Interv Aging. 2008;3:445-51.

25. Lee MS. Minimally invasive total hip arthroplasty. Formosan J Musculoskeletal Dis. 2011;2:41-4.

26. Chen D, Hu C, Chang Y, et al. Comparison of clinical outcome in primary total hip arthroplasty by conventional antero-lateral trans-gluteal or 2-incision approach. J Arthroplasty. 2009;24:528-32.

27. Engh CA, Massin P, Suthers KE. Roentgenographic assessment of the biologic fixation of porous-surfaced femoral components. Clin Orthop Relat Res. 1990;257:107-28.

28. Johnston RC, Fitzgerald Jr RH, Harris WH, et al. Clinical and radiographic evaluation of total hip replacement. A standard system of terminology for reporting results. J Bone Joint Surg Am. 1990;72:161-8.

29. Bodén $H$, Adolphson $P$. No adverse effects of early weight bearing after uncemented total hip arthroplasty: a randomized study of 20 patients. Acta Orthop Scand. 2004;75:21-9.

30. Kamiya N, Suzuki $\mathrm{H}$, Endo $\mathrm{T}$, et al. Clinical usefulness of bone markers in prostate cancer with bone metastasis. Int J Urol. 2012;19:968-79.

31. Savarino L, Granchi D, Cenni E, et al. Systemic cross-linked N-terminal telopeptide and procollagen I C-terminal extension peptide as markers of bone turnover after total hip arthroplasty. J Bone Joint Surg (Br). 2005;87:571-6.

32. Glassman AH, Bobyn JD, Tanzer M. New femoral designs: do they influence stress shielding? Clin Orthop Relat Res. 2006;453:64-74.

33. Eriksen EF, Lyles KW, Colon-Emeric CS, et al. Anti-fracture efficacy and reduction of mortality in relation to timing of the first dose of zoledronic acid after hip fracture. J Bone Miner Res. 2009;24:1308-13.

34. Reid IR, Black DM, Eastell R, et al. HORIZON Pivotal Fracture Trial and HORIZON Recurrent Fracture Trial Steering Committees, Reduction in the risk of clinical fractures after a single dose of zoledronic Acid 5 milligrams. J Clin Endocrinol Metab. 2013;98:557-63.

35. Black DM, Reid IR, Boonen S, et al. The effect of 3 versus 6 years of zoledronic acid treatment of osteoporosis: a randomized extension to the HORIZONPivotal Fracture Trial (PFT). J Bone Miner Res. 2012;27:243-54.

36. Mehrotra B, Ruggiero S. Bisphosphonate complications including osteonecrosis of the Jaw. Hematol Am Soc Hematol Educ Program. 2006;515:356-60.

37. Shane E, Burr D, Abrahamsen B, et al. Atypical subtrochanteric and diaphyseal femoral fractures: second report of a task force of the American Society for Bone and Mineral Research. J Bone Miner Res. 2014;29:1-23.

38. Unnanuntana A, Saleh A, Mensah KA, et al. Atypical femoral fractures: what do we know about them? AAOS Exhibit Selection. J Bone Joint Surg Am. 2013;95:1-13. e8.

\section{Submit your next manuscript to BioMed Central and we will help you at every step:}

- We accept pre-submission inquiries

- Our selector tool helps you to find the most relevant journal

- We provide round the clock customer support

- Convenient online submission

- Thorough peer review

- Inclusion in PubMed and all major indexing services

- Maximum visibility for your research

Submit your manuscript at www.biomedcentral.com/submit
Biomed Central 